\title{
Multi-source Motion Decoupling Ablation Catheter Guidance for Electrophysiology Procedures
}

\author{
Mihaela Constantinescu ${ }^{1}$, Su-Lin Lee ${ }^{1}$, Sabine Ernst ${ }^{2}$, and Guang-Zhong Yang ${ }^{1}$ \\ 1 The Hamlyn Centre for Robotic Surgery, Imperial College London, London, UK \\ ${ }^{2}$ The Royal Brompton and Harefield Hospital, London, UK \\ mihaela.constantinescu12@imperial.ac.uk
}

\begin{abstract}
Accurate and stable positioning of the ablation catheter tip during the delivery of radiofrequency impulses in cardiac electrophysiology remains a challenge due to the endocardium motion from multiple sources (cardiac cycle and respiration) and inevitable slippage of the catheter tip. This paper presents a novel ablation catheter guidance framework during electrophysiology procedures. Catheter tip electrode position readings from intraoperative electroanatomical data are used to decouple tip motion from different motion sources as part of the preablation mapping. The resulting information is then used to determine if there is relative slippage between the catheter tip and endocardial surface and is shown as a probability map for online decision support of the ablation process. The proposed decomposition method and the slippage assessment were performed on a retrospective cohort of 19 patients treated for ventricular tachycardia (13 cases) or atrial fibrillation (6 cases) and were also validated on artificially generated signals.
\end{abstract}

\section{Introduction}

Cardiac rhythm disorders are a major cause of sudden death worldwide, with atrial fibrillation and ventricular tachycardia being the most prevalent [1]. Globally, atrial fibrillation alone affects 33.5 million people and its related deaths doubled between 1990 and 2010 [2]; nevertheless, many patients are treated before the condition becomes critical.

One of the treatment options available is the ablation of the sources or paths for ectopic impulses under guidance from the CARTO system (Biosense Webster, Diamond Bar, CA, USA) which gives electroanatomical information from reference and mapping/ablation catheters, triggered at sparse points in the cardiac cycle. However, each patient still requires an average of 1.3 procedures with a $70 \%$ success rate [3], among the causes being the lack of patient-specific dynamic guidance for both the scar development during ablation and the cardiac wall motion at the desired ablation point [4]. The motion of the cardiac wall, as well as the blood flow in the endocardium near the vessel wall, can cause slippage of the ablation catheter tip which can be over $12 \mathrm{~mm}$ in $2.5 \mathrm{~s}$ (Fig. 1a), 
as measured from electroanatomical data used in this work. Such slippage may result in ablation lesions not originally planned by the interventional cardiologist and in repeated procedures for the patient. Although there are certain procedures where sliding along the endocardium is desired during ablation, the present study assumes focused ablation of an endocardial point.
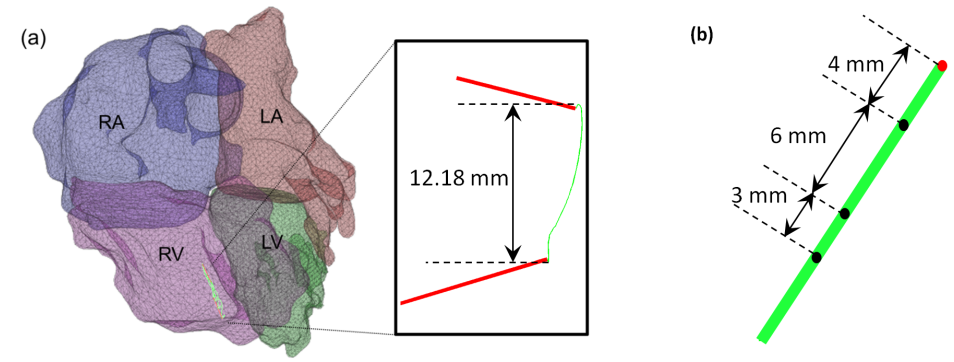

Fig. 1. (a) The cardiac chambers (RA - right atrium, RV - right ventricle, LA - left atrium, LV - left ventricle) with the catheter placed in the RV. Absolute catheter tip motion can be as high as $12 \mathrm{~mm}$. The red catheter shapes indicate the motion range and the green curve is the trajectory of the ablation catheter tip. (b) Spatial distribution of the mapping and ablation (MA) catheter sensor and electrodes.

Several approaches have been investigated to estimate the motion of the catheter tip intraoperatively. Principal Component Analysis (PCA) has been applied on the reference catheter shape vectors extracted from fluoroscopy images [5]. Other image-based approaches proposed include 2D tracking of the interventional reference catheters [6], which was also extended to 3D under epipolar constraints [7]. While these methods are able to track the motion, they depend on X-ray fluoroscopy, whose use is avoided due to radiation exposure.

The respiratory and cardiac motions have also been estimated from 3D electroanatomical mapping datasets. Roujol et al. [8] simplified the motion model by removing the two signals from fixed frequency bands after analysing the catheter tip position in the Fourier domain. Another recent approach fitted a model of motion to the catheter tip by instantiating a bilinear atlas of patient-specific shapes and deformations [9]. These methods, however, need a good spread of the recorded points on the endocardium in order for the instantiation to have a small error.

This paper proposes a method for online assessment of ablation catheter tip contact with the endocardium based on recordings of catheter tip position. A probabilistic slippage map of the catheter tip with respect to the endocardium is provided for online guidance of the ablation catheter, allowing the operator to adjust the catheter, should slippage occur. Validation of the proposed method was performed on 19 data sets from patients treated for ventricular tachycardia and atrial fibrillation, as well as on artificially generated mixed signals. 


\section{Methods}

\subsection{Data Acquisition}

Simulated data with known ground truth. Artificial data sets of 3D signals with their first order derivatives were generated in order to simulate 29 respiratory frequencies, $(12 \ldots 40) \mathrm{min}^{-1}$, and 96 different starting points of the recording relative to the respiratory cycle length, i.e. 96 different phase shifts, (0...95) \%. It was assumed that the cardiac and respiratory components follow phase-shifted sine waves with amplitudes of 5 and $20 \mathrm{~mm}$, respectively. A linear drift of $2 \mathrm{~mm} / \mathrm{s}$, accounting for a slip of $5 \mathrm{~mm}$ in $2.5 \mathrm{~s}$, was added to the 3D signal. No noise was added, as the real signals were smoothed with an average filter in the preprocessing step of the decomposition method.

Patient studies. For the patient studies, data from a Navistar MA catheter were used (Fig. 1b). The sensor at the tip records the electrocardiogram (ECG) as well as the 3D position. Additionally, the 3D positions of the other four electrodes on the MA catheter and the first order derivatives of all signals were used for motion decoupling. All signals were linearly interpolated to generate 2500 time stamps. The intraoperative data comprised 19 studies from 13 ventricular tachycardia and 6 atrial fibrillation patients, each with a different number of mapping points. The ECG recordings were used to remove the mapping points acquired during arrhythmic episodes, i.e. cardiac period under $600 \mathrm{~ms}$, as it was assumed that the ablation will be performed during episodes of normal heart beat so that the contact with the wall is more easily maintained. The number of normal sinus mapping points per study ranged from 10 to 63 .

\subsection{Motion Decoupling}

Multivariate empirical mode decomposition (MEMD) [11] was used to decouple the three-dimensional position signals of the MA catheter sensor and electrodes. The method sorts the zero-mean components, called intrinsic mode functions (IMF), in descending order of their number of local extrema, which in the case of periodic events, such as cardiac and respiratory displacement, is equivalent to sorting them by the signal frequency (amplitude and frequency modulation of the mixed input signal).

In the original empirical mode decomposition (EMD) [10], the IMFs have a physical meaning in the original mixed EEG signal. Analogously, the component with the least number of local extrema showing the general trend in the signal must have a physical correspondence in the relative slippage. The next in periodicity was identified as the respiration, followed by the cardiac motion. Eq. (1) shows the composition of the mixed signal assumed by the MEMD algorithm, with $\mathbf{x}, \mathbf{y}$, and $\mathbf{z}$ being the original signals from the sensor and the four electrodes over $2.5 \mathrm{~s}, \mathrm{M}$ the number of decomposed IMFs, $\mathbf{a}_{m, k}$ the amplitude value which modulates the oscillation vector $\boldsymbol{\Psi}_{m}$ of the $m$-th IMF at time stamp $k$, and $\mathbf{r}$ the residual trend vector for each channel. 


$$
\left[\begin{array}{l}
\mathbf{x}_{k} \\
\mathbf{y}_{k} \\
\mathbf{z}_{k}
\end{array}\right]=\sum_{m=1}^{\mathrm{M}} \mathbf{a}_{m, k} \boldsymbol{\Psi}_{m, k}+\mathbf{r}_{k}, \quad k=1 . .2500
$$

The simultaneous decomposition with MEMD as compared to the singlechannel EMD ensures not only the same number $\mathrm{M}$ of independent IMFs in all channels, but also their alignment in the frequency domain. EMD, the singlechannel decomposition method, for a variable $x$ can be summarised in the following steps $[10,12]$ :

1. Find the local extrema of $x$.

2. Perform spline interpolation between the local minima and local maxima, respectively, thus generating two envelope signals.

3. Calculate the mean $m(t)$ of the two envelopes.

4. Subtract $m(t)$ from $x(t)$ to obtain a candidate for IMF. $d(t)=x(t)-m(t)$ is an IMF if it has a zero mean and the number of zero crossings and extrema are equal or differ by one.

5. If $d(t)$ does not satisfy these two criteria, $x(t)=d(t)$ and reinitialise the algorithm.

6. If $d(t)$ is a valid IMF, $x(t)=x(t)-d(t)$ and restart the algorithm.

7. Run the algorithm until $x(t)$ (the residual) becomes a monotonic function.

The multivariate extension computes the mean in the multidimensional space by first generating a Hammersley sequence for projecting the 30-dimensional sequence (one sensor and four electrodes, each with three dimensions and their derivatives) onto certain directions. The means are calculated for the projection signals and then subtracted as in the one-dimensional algorithm [11].

\subsection{Probabilistic Model}

A Dynamic Bayesian Network was used to model the conditional dependency of the ablation catheter tip (Tip) on the cardiac motion $(\mathrm{C})$, respiration $(\mathrm{R})$, and slippage $(\mathrm{S})$, in a probabilistic framework (Fig. 2). In a single step, Tip was the observed variable as measured by the electromagnetic position sensors, while the other variables were extracted using MEMD. P (S|Tip,C,R) in an intercausal reasoning approach and the interstate dependencies $\mathbf{P}\left(\mathrm{R}^{\prime} \mid \mathrm{S}, \mathrm{R}\right)$ and $\mathbf{P}\left(\mathrm{C}^{\prime} \mid \mathrm{S}, \mathrm{C}\right)$ were computed from a Gaussian Mixture Model trained on the slippage and the MA catheter tip position.

\section{Results}

Simulated data with known ground truth. Results from the artificial data sets are shown in Fig. 3. Both amplitude and frequency can be recovered in the respiratory signal with an error of under $10 \%$ in the case of a respiratory rate between 12 and $30 \mathrm{~min}^{-1}$, which is the normal respiratory rate in adults. The starting point of the recording interval relative to the respiratory cycle does not influence the recovery. The cardiac signal extraction for these cases yielded a mean amplitude error of $9.33 \%$ and a mean frequency error of $11.73 \%$. 


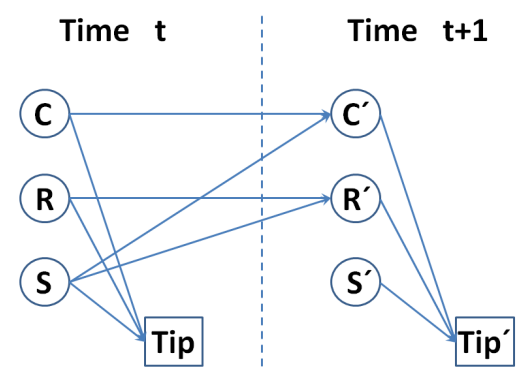

Fig. 2. Conditional dependency of the ablation catheter tip (Tip) on the cardiac motion (C), the respiration (R), and the slippage (S). Squared variables are observed, circled variables are unknowns. $\mathbf{P}(\mathrm{S} \mid \mathrm{Tip}, \mathrm{C}, \mathrm{R})$ is sought.

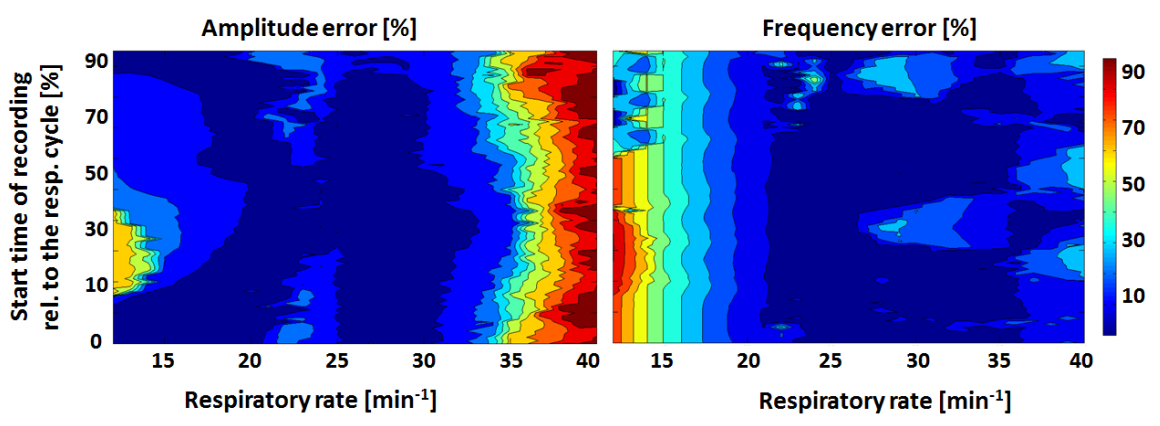

Fig. 3. MEMD respiration recovery using simulated data with known ground truth. The jagged contours indicate that there are some combinations of phase shift and respiratory rate for which the decomposition is either slightly better or worse than the trend.

Patient studies. MEMD was applied to each sinus mapping point of the 19 studies. Fig. 4 shows the original catheter tip position recording, followed by the relative slippage and the respiratory and cardiac components for each axis and for four different points around a mapped right ventricle. The slippage accounted for the biggest motion of the catheter tip, exhibiting as a constant drift in the least periodic IMF. The second component was the respiratory motion, identified as the IMF next in frequency. Finally, the third factor was classified as the cardiac component due to its periodicity compared to the ECG. The relative error in the estimated heart rate (HR) was assessed (Fig. 5).

A Gaussian Mixture Model was trained on each set of mapping points, giving the conditional dependency $\mathbf{P}(\mathrm{S} \mid \mathrm{Tip}, \mathrm{C}, \mathrm{R})$ as a normal distribution. Fig. 5 gives an overview of several study-specific values, including the maximum absolute slip in $\mathrm{mm}$ and the probability of this maximal relative slip. The heart rate error was higher for faster ECGs, at the arrhythmic border of the $600 \mathrm{~ms}$ period. In addition, two other probabilities were computed from the conditional dynamic 

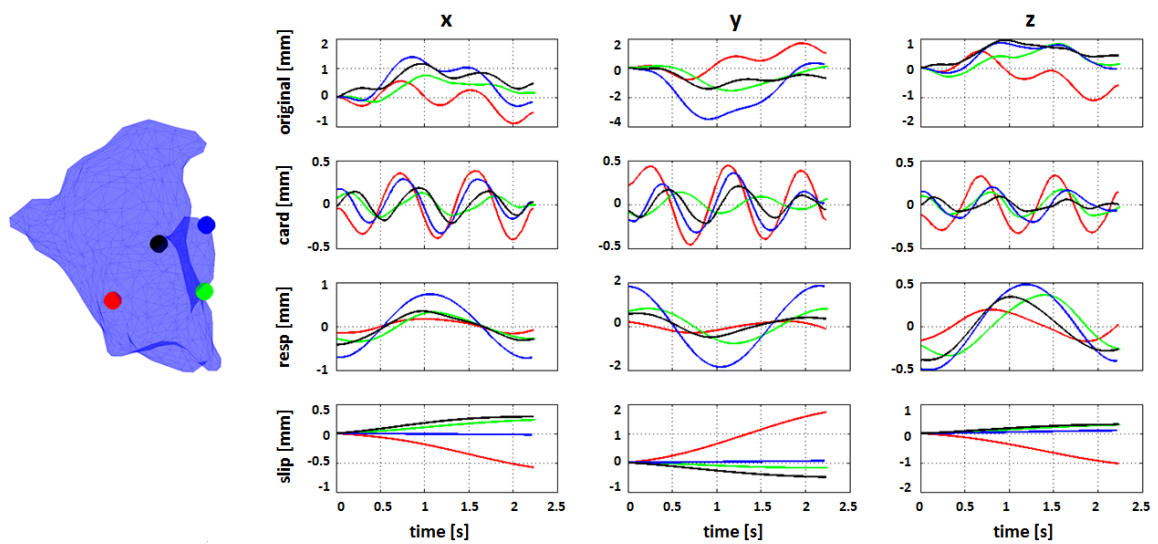

Fig. 4. Components extracted from 4 mapping points in one ventricle and the location of the points within the anatomy.

network, estimating the recovery of the cardiac and respiratory motion after a slip of the catheter tip to a new endocardial point moving on different cardiac $\left(\mathrm{C}^{\prime}\right)$ and respiratory (R') waves.

Finally, Fig. 6 shows a visual indication of the probability of slippage at the mapping points in contact with the catheter tip when the electrode position and the cardiac and respiratory components are known. Additionally, the maximal expected slippage is shown. This combined information is able to provide the operator with online decision support of the ablation process and may influence the adjustment of the ablation point, force, or the use of robotic catheter stabilisation.

\section{Discussion and Conclusion}

MEMD provides an initial solution to the source decomposition problem in a simplified scenario of intracardial motion due to its suitability to decouple periodic signals from monotonic drifting trends. However, because of the short acquisition time of $2.5 \mathrm{~s}$ for each mapping point, the MEMD algorithm failed to decompose the signal into three clear physiological components when the respiratory sequence was a monotonic function. In this case, the method added the respiratory motion in the observed time interval to the monotonic relative slippage. Out of 517 sinus mapping points analysed with the decomposition method, in 183 cases the respiration could not be decoupled from the relative slippage. The outcome can be potentially improved with respiratory gating or by adding a full-period respiratory sequence as an additional channel to the algorithm.

Nevertheless, the source separation provided a good starting point for the novel probabilistic guidance. With an average of 27 mapping points per study, the trained Gaussian Mixture Model computed a reliable distribution of condi- 


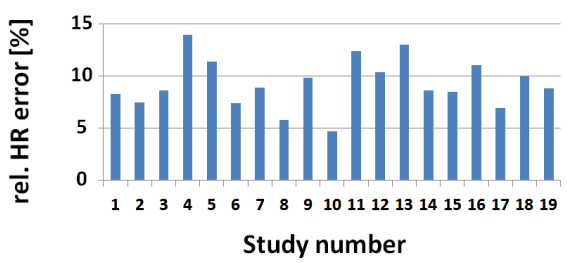

(a)

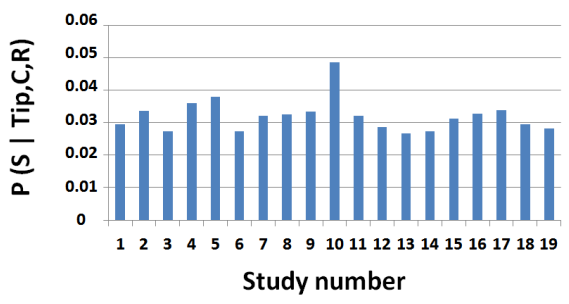

(c)

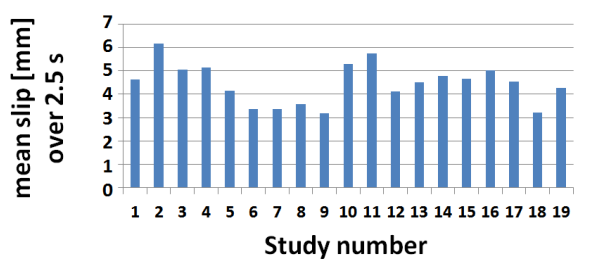

(b)

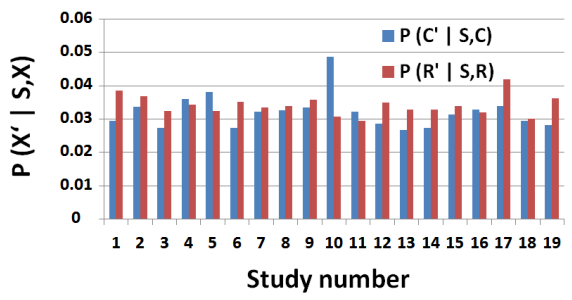

(d)

Fig. 5. (a) Relative heart rate error of the recovered cardiac motion compared to ECG, (b) Mean relative tip motion with respect to the endocardium, (c) Probability of slippage given the tip position, the cardiac motion and the respiration, (d) Probabilities of recovering the cardiac and respiratory components after slippage from the old endocardium point with a C-R motion to a new endocardium point C'-R'.
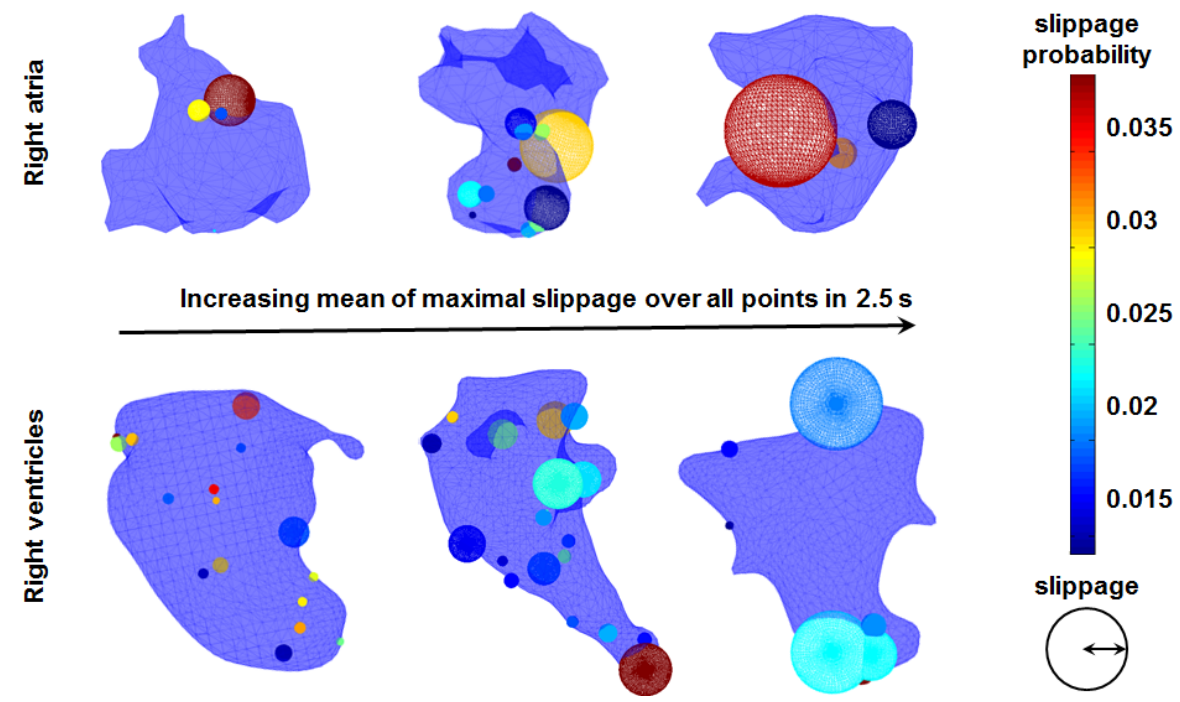

Fig. 6. Example of probabilistic maps of slippage with the radius of the sphere showing the maximum slippage computed over $2.5 \mathrm{~s}$. The sphere is a real-size expectancy of relative slippage which may occur with the probability coded in the colour. 
tional dependencies. Significant information could be gained from the intercausal inference based on the known cardiac motion and respiration, i.e. $\mathbf{P}(\mathrm{S} \mid \mathrm{Tip}, \mathrm{C}, \mathrm{R})$.

In conclusion, a new method for the assessment of intracardiac ablation catheter motion by incorporating probabilistic estimation of the relative slippage from the moving endocardium was proposed. The method also provides a new intraoperative visualisation tool to guide the surgeon during the ablation, to facilitate the stabilisation of the catheter tip in a robotic framework, and finally to select the optimal ablation point with a lower probability of drift over the planned ablation period.

\section{Acknowledgments}

We thank Professor Danilo Mandic, Communication and Signal Processing Research Group, Department of Electrical and Electronic Engineering, Imperial College London, for the code for Multivariate Empirical Mode Decomposition and for his guidance in the application thereof.

\section{References}

1. Fuster V, Ryden LE, Cannom DS, et al., ACC/AHA/ESC 2006 Guidelines for the Management of Patients with Atrial Fibrillation: A Report of the American College of Cardiology/American Heart Association Task Force on Practice Guidelines and the European Society of Cardiology Committee for Practice Guidelines, Circulation. 2006;114(7):e257-354

2. Chugh S, Havmoeller R, Narayanan K, et al., Worldwide Epidemiology of Atrial Fibrillation: A Global Burden of Disease 2010 Study, Circulation. 2014;129:837-847

3. Cappato R, Calkins H, Chen SA, et al., Updated worldwide survey on the methods, efficacy, and safety of catheter ablation for human atrial fibrillation, Circulation. $2010 ; 3: 32-38$

4. Klemm H, Steven D, Johnsen C, et al., Catheter motion during atrial ablation due to the beating heart and respiration: impact on accuracy and spatial referencing in three-dimensional mapping, Heart Rhythm. 2007;4(5):587-592

5. Panayiotou M, King AP, Ma Y, et al., Statistical model of catheter motion from interventional x-ray images: application to image-based gating, Phys. Med. Biol. 2013;58:7543-7562

6. Brost A, Liao R, Hornegger J, Strobel N, Model-based registration for motion compensation during EP ablation procedures, WBIR, LNCS. 2010;234-245

7. Ma Y, Gao G, Gijbers G, et al., Image-based automatic ablation point tagging system with motion correction for cardiac ablation procedures, IPCAI, LNCS. 2011;145155

8. Roujol S, Anter E, Josephson M, Nezafat R, Characterization of respiratory and cardiac motion from electro-anatomical mapping data for improved fusion of MRI to left ventricular electrograms, PLoS ONE. 2013;8(11): e78852

9. Porras AR, Piella G, Berruezo A, et al., Interventional endocardial motion estimation from electroanatomical mapping data: application to scar characterization, Transactions on Biomedical Engineering. 2013;60(5):1217-1224 
10. Huang N, Shen Z, Long S, et al., The empirical mode decomposition and the Hilbert spectrum for nonlinear and non-stationary time series analysis, Proc. R. Soc. Lond. 1998;454:903-995

11. Rehman N, Mandic DP, Multivariate empirical mode decomposition, Proc. R. Soc. 2010;466:1291-1302

12. Mandic DP, Rehman N, Wu Z, Huang N, Multivariate empirical mode decomposition, IEEE Signal Processing Magazine November 2013;74-86 